\title{
SOME RESULTS ABOUT THE KAUFFMAN BRACKET SKEIN MODULE OF THE TWIST KNOT EXTERIOR
}

\author{
RĂZVAN GELCA AND FUMIKAZU NAGASATO
}

\begin{abstract}
In this paper, we list in explicit form the factoring relations of the Kauffman bracket skein module (KBSM for short) of a twist knot exterior. This is done using curves decorated by characters of irreducible $S L(2, \mathbb{C})$-representations. In the process, we exhibit a relation which holds in the KBSM of the knot exterior, called the minimal relation. In the final section we prove that, when specializing the variable of the Kauffman bracket at $t=-1$, the minimal relation becomes the defining polynomial of the $S L(2, \mathbb{C})$-character variety of the twist knot.
\end{abstract}

\section{Motivation AND BACKGRound}

1.1. Motivation. The Kauffman bracket skein module of a 3-manifold was introduced by J. Przyticki as a natural generalization of the Kauffman bracket to general 3 -manifolds. It was later linked to the character variety of $S L(2, \mathbb{C})$-representations of the fundamental group of the manifold [B1], [PS]. In this perspective, Kauffman bracket skein modules were used to give an alternate description of the A-polynomial of Cooper, Culler, Gillet, Long, and Shalen, and to generalize it to a noncommutative setting [FGL]. The computation of the noncommutative generalization of the A-polynomial of a knot relies heavily on the good understanding of the skein module of the knot complement.

The noncommutative generalization of the A-polynomial was computed for the unknot in [FGL], trefoil knot in [G1], partially for $(2,2 p+1)$-torus knots in [GS1], and for the figure-eight knot in [GS2]. In those papers, and also in [G2] this knot invariant was linked to the Jones polynomial. This relation led to new developments in the study of colored Jones polynomials [GL]. Let us also point out that such computations yield an alternative way of finding the classical A-polynomial, as it was discussed in $[\mathrm{N}]$.

The computation itself is done in three stages. The first stage consists of the understanding of the Kauffman bracket skein module of the knot complement. As knot complements can be obtained by attaching 2-handles to a handlebody, and such topological operations yield algebraic factorizations at the level of the skein module, it is necessary to write the factoring relations in explicit form. The second stage of the computation is concerned with determining the action of the Kauffman bracket skein algebra of the torus on the skein module of the knot complement, while the third stage is about finding the annihilator of the empty skein.

The present paper describes the first step in the computation of the noncommutative version of the A-polynomial for twist knots. Our convention is to count twists

Key words and phrases. A-polynomial, colored Kauffman brackets, Kauffman bracket skein module, tunnel number.

The second author has been supported by JSPS Research Fellowships for Young Scientists. 
as in Figure 1 (which some authors call half-twists). We will also be concerned only with the case of positive twists. The Kauffman bracket skein module of the complement of a twist knot was determined in [BL]. Let us mention that recently T. Le extended this result to all 2-bridge knots [L]. However, none of the above mentioned works gives an explicit description of the factoring relations in terms of the basis of the skein module. This is the purpose of the present paper.

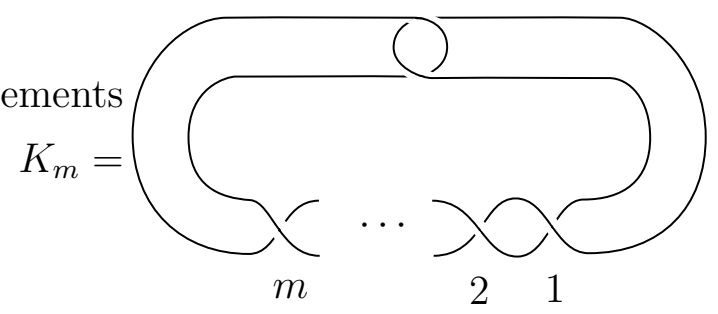

Figure 1 . The $m$-twist knot $K_{m}$ : the twists are ordered from the right to the left

\subsection{The Kauffman bracket skein module of the $m$-twist knot exterior.} Denote by $K_{m}$ the $m$-twist knot $(m \geq 0)$ in $S^{3}$, by $E_{K_{m}}$ its exterior (i.e. the complement in $S^{3}$ of a regular neighborhood). Bullock and Lofaro have proved the following result.

Theorem 1 (Bullock, Lofaro [BL]). The Kauffman bracket skein module of the complement of a regular neighborhood of the $m$-twist knot is the free $\mathbb{C}\left[t, t^{-1}\right]$-module with basis $x^{i} y^{j}, i \geq 0,0 \leq j \leq m$, where $x$ and $y$ are the curves described in Figure 2 .

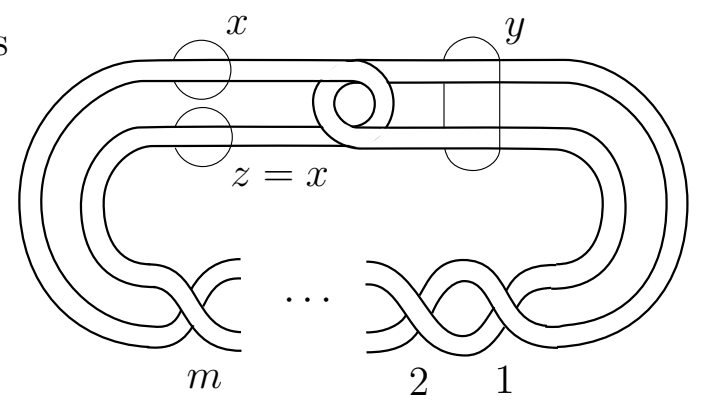

Figure 2. Skeins $x, y$ and $z$ in $\mathcal{K}_{t}\left(E_{K_{m}}\right)$

Let us recall briefly the idea of the proof. Because the tunnel number of $K_{m}$ is 1, the knot exterior $E_{K_{m}}$ can be obtained by attaching a 2-handle to the genus 2 handlebody $\mathrm{H}_{2}$.

The proof relies on the following theorems.

Theorem 2 (Przytycki [P2]). The KBSM of the genus 2 handlebody is the free $\mathbb{C}\left[t, t^{-1}\right]$-module with basis $x^{i} y^{j} z^{k}, i, j, k \in \mathbb{Z}_{\geq 0}$, where $x, y$ and $z$ are the curves from Figure 3. 


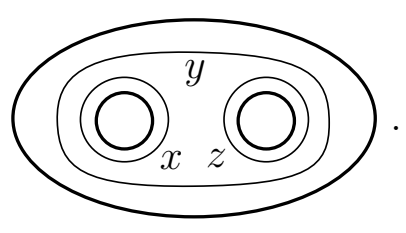

Figure 3. Skeins $x, y$ and $z$ in $\mathcal{K}_{t}\left(H_{2}\right)$

Theorem 3 (Przytycki [P1]).

$$
\mathcal{K}_{t}\left(E_{K}\right)=\mathcal{K}_{t}\left(H_{2}\right) / J
$$

where $J$ is the submodule of $\mathcal{K}_{t}\left(H_{2}\right)$ generated by

$$
\left\{L-\operatorname{sl}(L) \mid L \text { : any framed link in } H_{2}\right\} .
$$

Here $\operatorname{sl}(L)$ means the link obtained from $L$ by an (arbitrary) handle slide through the attached 2-handle.

We see immediately that in $\mathcal{K}\left(E_{K_{m}}\right)$ the skein $z$ is identified with the skein $x$ as seen in Figure 2 (via a handle slide relation).

The work of Bullock and Lofaro is concentrated on the elimination of the higher powers of $y$ using handle-slides. In our paper we will give explicit formulas for these relations. These formulas are necessary for computing the action of the Kauffman bracket skein algebra of the boundary torus, and ultimately they should lead to computation of the noncommutative A-ideal of a twist knot.

\section{The EXPLicit FORM OF THE FACTORING RELATIONS}

It is important to observe that, as it was the case with previously studied knots [G1] [GS1], the characters of irreducible $S L(2, \mathbb{C})$-representations play a special role. For this reason we will change the basis of the skein module of the knot exterior. Recall that $S_{n}(x), n \geq 0$ are the polynomials defined recursively by $S_{0}(x)=1$, $S_{1}(x)=x$, and $S_{n+1}(x)=x S_{n}(x)-S_{n-1}(x)$, definition extended for all integers $n$. These are the Chebyshev polynomials of second type.

The basis of the Kauffman bracket skein module of the twist knot exterior that we prefer is

$$
\left\{S_{i}(x) S_{j}(y), \quad 0 \leq i, 0 \leq j \leq m\right\} .
$$

Let us remark that for a curve $c, S_{n}(c)$ is the curve colored by the $n$th Jones-Wenzl idempotent.

2.1. The "minimal relation" in the KBSM of the twist knot exterior. There is a special curve in the handlebody which can be used to obtain all the necessary factorization relations. Sliding this curve through the 2-handle yields a relation which we call the minimal relation.

Figure 4 describes the attaching curve. In this figure the handlebody is obtained by drilling through a ball two "tunnels" that twist around each other. A handle slide consists of a band sum with the attaching curve.

We now make an important convention. There is a handle-slide that identifies the curves $x$ and $z$. We impose this condition in the skein module of the handlebody, 
i.e. we factor the skein module by the relation $x=z$. Everything below is done in this hypothesis.

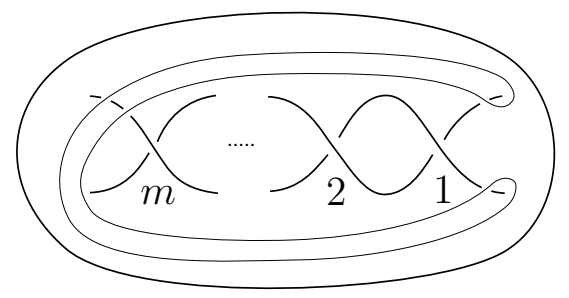

FiguRE 4. Attaching slope of $H_{2} \cup(2$-handle): Thick lines represent holes through a ball. The thin curve is the attaching slope; it lies on the boundary of $H_{2}$.

Consider the skeins $X_{i}$ in $\mathcal{K}_{t}\left(H_{2}\right)$ defined as follows:

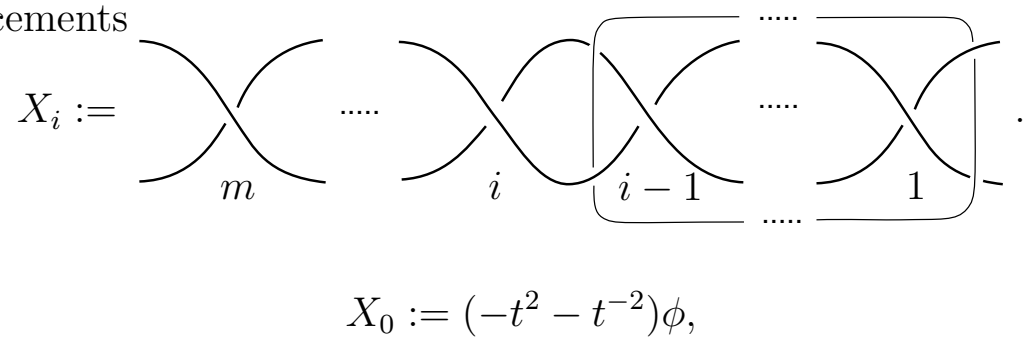

where $\phi$ denote the empty link. The skein $X_{m+1}$ is the one that gives the minimal relation.

Lemma 1 (Recursive relation of $\left.X_{i}\right)$. The skein $X_{i},(m+1 \geq i \geq 0)$, as an element in $\mathcal{K}_{t}\left(E_{K_{m}}\right)$ satisfies the following recursive relation:

$$
X_{i+2}-t^{2} y X_{i+1}+t^{4} X_{i}+2 t^{2} x^{2}=0, X_{1}=-t^{2} x^{2}-t^{4} y, X_{0}=-t^{2}-t^{-2} .
$$

Proof. All of the operations below are done in the handlebody $\mathrm{H}_{2}$. First, transform $X_{i+1}$ as follows:
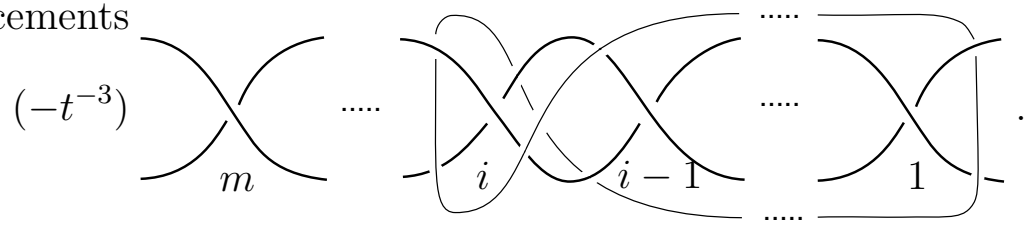

Then slide the kink to the right side as below:

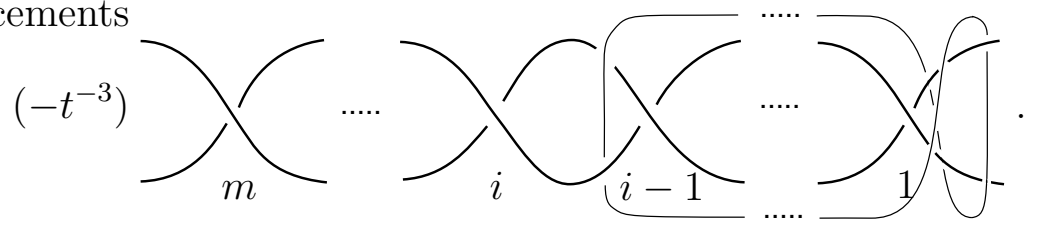


Here resolve the crossing by using the skein relation. For example,
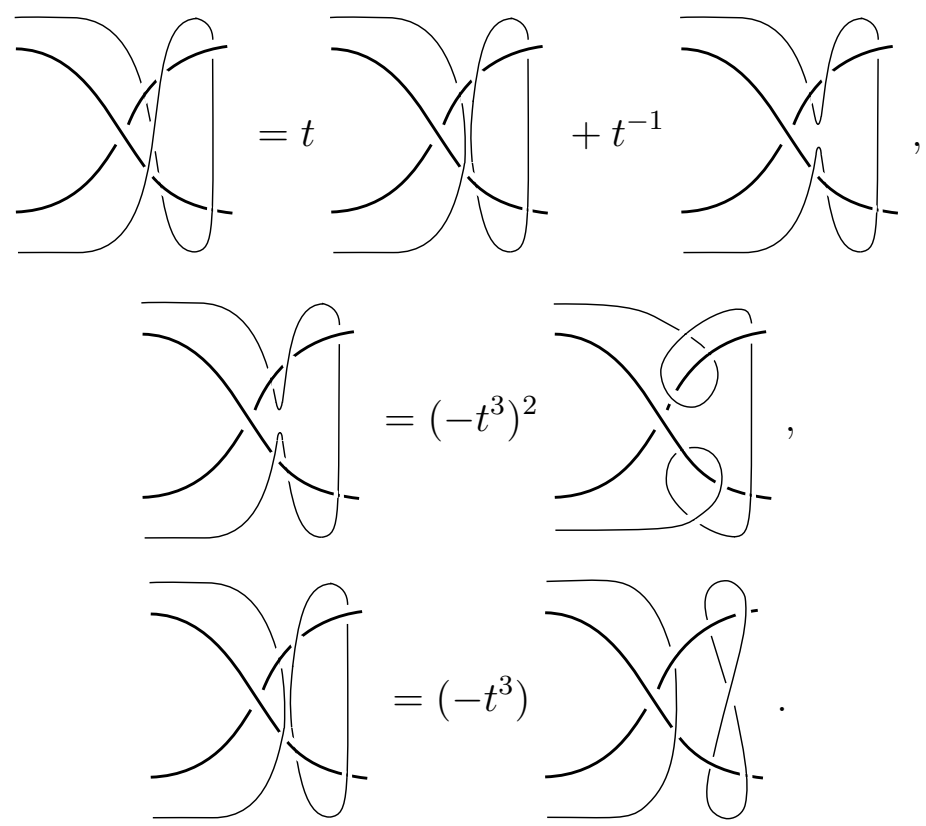

Substituting $z=x$ (which is allowed according to our convention), we can get the recursive relation stated in Lemma 1.

Lemma 2 (General term). The skeins $X_{i},(0 \leq i \leq m+1)$ can be written in terms of the basis of the skein module as

$$
\begin{aligned}
X_{i} & =-t^{2(i+1)} S_{i}(y)-t^{2 i} S_{i-1}(y) x^{2}+t^{2(i-1)} S_{i-2}(y)-2 t^{2(i-1)} x^{2} \sum_{n=0}^{i-2} t^{2 n} S_{n}(y) \\
& =-t^{2(i+1)} S_{i}(y)-t^{2 i} S_{i-1}(y) x^{2}+t^{2(i-1)} S_{i-2}(y)-2 x^{2} \frac{t^{2(i-1)} S_{i-1}(y)-t^{2 i} S_{i-2}(y)-1}{y-t^{2}-t^{-2}},
\end{aligned}
$$

There is no actual fraction in the second expression, as a polynomial in $y$, the numerator is divisible by the denominator. It is a matter of convenience to write it this way.

Proof. According to Lemma 1 the $X_{i}$ 's satisfy a second order nonhomogeneous recursive relation with constant coefficients. Set $a_{i}=t^{-2 i} X_{i}$, so that the homogeneous part of the recursive relation looks like $a_{i+1}-y a_{i}+a_{i-1}=0$, which is that of the Chebyshev polynomials. The general term is of the form $\alpha S_{i}(y)+\beta S_{i-1}(y)$, since $S_{i}(y)$ and $S_{i-1}(y)$ form a basis for the (2-dimensional) space of sequences satisfying this recursive relation.

On the other hand, the nonhomogeneous term is constant, so we can use the method of undetermined coefficients to find a particular solution, which is $-2 x^{2} /(y-$ $t^{2}-t^{-2}$ ) (just formally). The coefficients $\alpha$ and $\beta$ are determined from the initial condition. Initially they appear as fractions, but a routine computation with Chebyshev polynomials produce the formulas from the statement.

Lemma 3 (Handle-slide relation). The handle-slide of $X_{m}$ yields the following relation

$$
X_{m+1}+t^{-4} X_{m}+t^{-2} x^{2}=0
$$


Proof. All of the operations below are done in the handlebody $H_{2}$ (modulo $x=z$ ). First, consider the following band sum of $X_{m+1}$ and the attaching slope along a band $b$ :

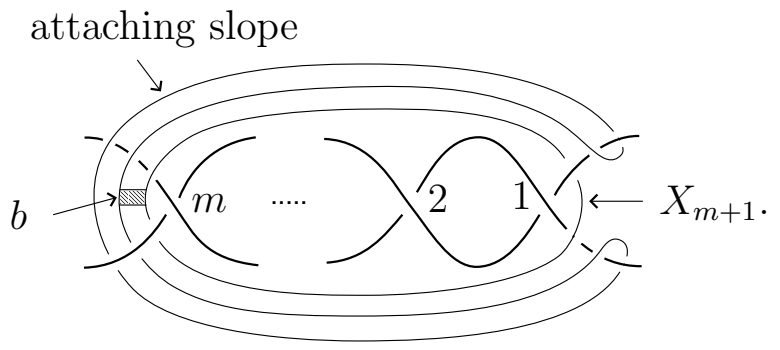

Let $s l_{b}\left(X_{m+1}\right)$ be the resulting knot after the band sum. Then a relation $s l_{b}\left(X_{m+1}\right)-$ $X_{m+1}=0$ holds in $\mathcal{K}_{t}\left(E_{K_{m}}\right)$. Resolving $s_{b}\left(X_{m+1}\right)$ and substituting $z=x$, we get,

$$
s l_{b}\left(X_{m+1}\right)=-t^{-4} X_{m}-t^{-2} x^{2} .
$$

This completes the proof.

Lemmas 2 and 3 give us the following theorem.

Theorem 4 (Minimal relation). Let

$$
\begin{aligned}
R_{m}(t):= & S_{m+1}(y)+\left(t^{-6}-t^{-2} x^{2}\right) S_{m}(y)+\left(\left(2 t^{4}+t^{-8}\right) x^{2}-t^{-4}\right) S_{m-1}(y)-t^{-10} S_{m-2}(y) \\
& +2 x^{2}\left(t^{-2 m-2}+t^{-2 m-6}\right) \sum_{i=0}^{m-2} t^{2 i} S_{i}(y)-t^{-2 m-6} x^{2} .
\end{aligned}
$$

Then in the complement of the m-twist knot $R_{m}(t)=0$.

The equation $R_{m}(t)=0$ is called the minimal relation.

2.2. The other factorization relations (The action of $S_{k}(y)$ on $X_{i}$ ). We now describe the relations that reduce $S_{m+k}(y), k \geq 2$ in terms of polynomials of lower degree.

We concentrate on the skeins $X_{i} * S_{k}(y), k \geq 0,0 \leq i \leq m+1$, defined as

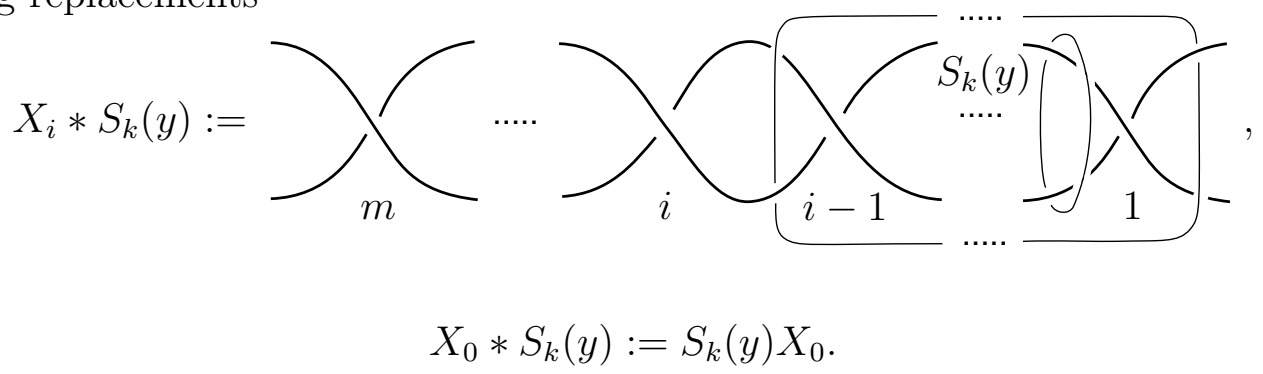

Lemma 4. For $k \geq 0$ and $i=1,2, \ldots, m+1$, as an element of $\mathcal{K}_{t}\left(E_{K_{m}}\right), X_{i} * S_{k}(y)$ satisfies the following relation:

$X_{i} * S_{k}(y)=t^{4} y\left(X_{i} * S_{k-1}(y)\right)+\left(-t^{6}+t^{-2}\right)\left(X_{i-1} * S_{k-1}(y)\right)-X_{i} * S_{k-2}(y)+2\left(-t^{4}+1\right) x^{2} S_{k-1}(y)$. 
Proof. First, consider the following configuration of $X_{i} *\left(y S_{k-1}(y)\right)$.

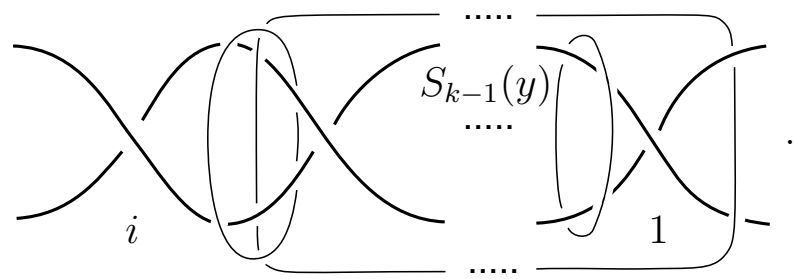

Calculate $X_{i} *\left(y S_{k-1}(y)\right)$ as $\left(X_{i} * y\right) * S_{k-1}(y)$. Resolving $X_{i} * y$ we can reduce $\left(X_{i} * y\right) * S_{k-1}(y)$ to

$$
\left\{t^{4} y X_{i}+\left(-t^{6}+t^{-2}\right) X_{i-1}+2\left(-t^{4}+1\right) x^{2}\right\} * S_{k-1}(y) .
$$

Indeed,
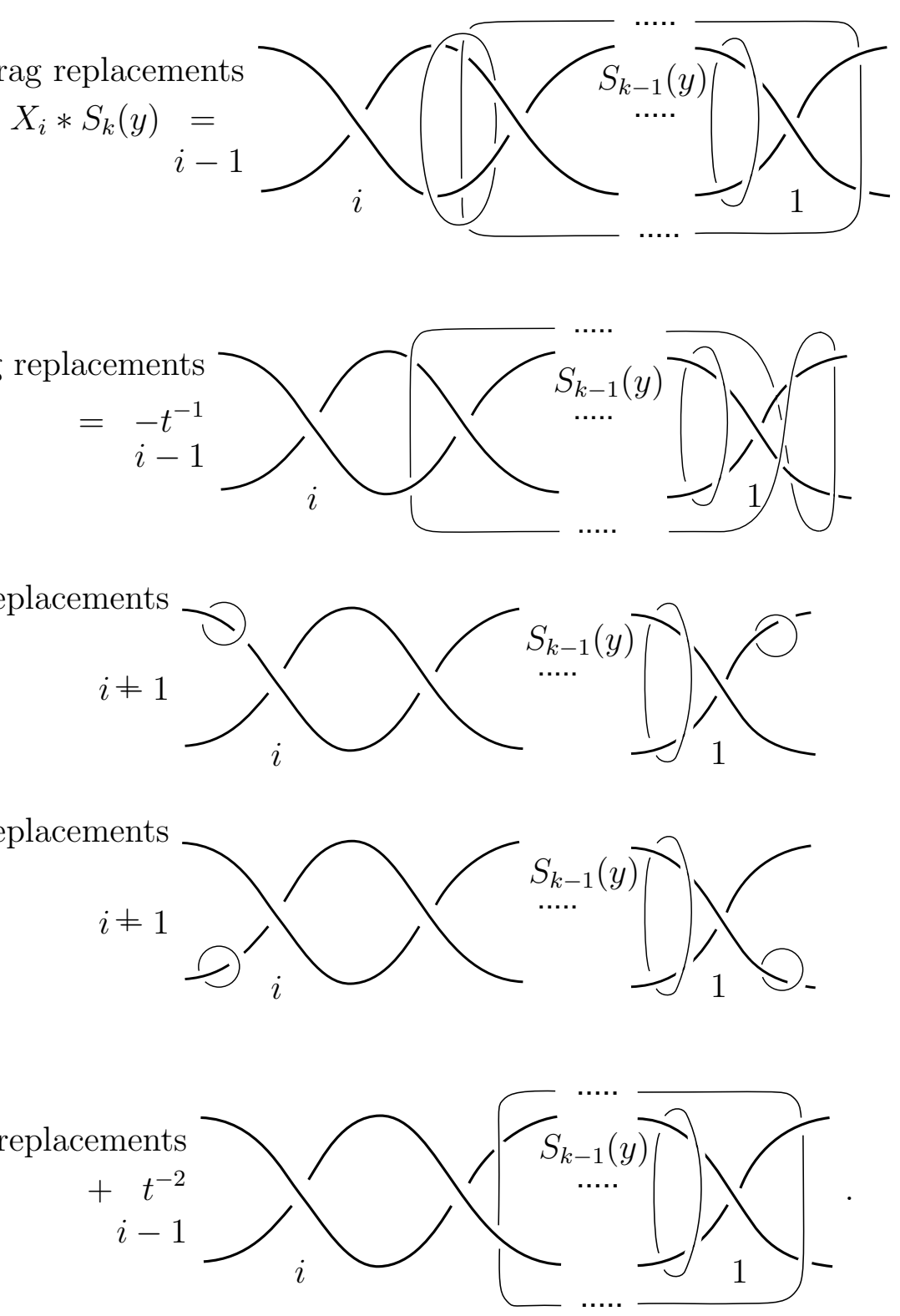
Note that we can calculate $\left(X_{i} * y\right) * S_{k-1}(y)$ separately as above, because there is no interaction between $X_{i} * y$ and $S_{k-1}(y)$. The term (2) can be calculated via the resolutions performed in the proof of Lemma 1 and is

$$
t^{4} y X_{i} * S_{k-1}(y)-t^{6} X_{i-1} * S_{k-1}(y)-2 t^{4} x^{2} S_{k-1}(y)
$$

Using the relation $y S_{k-1}(y)=S_{k}(y)+S_{k-2}(y)$, we obtain the desired recursive relation.

As a consequence, we have

Lemma 5 (General term).

$$
\begin{aligned}
t^{-2 i} X_{i} * S_{k}(y)= & \left(y-t^{2}-t^{-2}\right)^{-1} \times\left[-t^{4 k+2} S_{n+k+1}(y)+t^{-4 k-2} S_{n-k-3}(y)\right. \\
& +t^{4 k}\left(t^{4}-S_{2}(x)\right) S_{n+k}(y)+t^{-4 k}\left(-t^{-4}+S_{2}(x)\right) S_{n-k-2}(y) \\
& +t^{4 k-2}\left(-t^{-4}+\left(t^{4}-1\right) S_{2}(x)\right) S_{n+k-1}(y) \\
& +t^{-4 k+2}\left(t^{4}-\left(t^{-4}-1\right) S_{2}(x)\right) S_{n-k}(y) \\
& \left.+2 x^{2} t^{-2 n} S_{k}(y)+\sum_{i=-k+1}^{k-1} t^{4 i} \varphi_{i-k}(x) S_{n+i}(y)\right]
\end{aligned}
$$

where

$$
\varphi_{j}(x)= \begin{cases}\left(t^{4}-t^{-4}\right) S_{2}(x), & \text { if } j \text { is odd } \\ t^{6}-t^{-6}+\left(t^{2}-t^{-2}\right) S_{2}(x), & \text { if } j \text { is even }\end{cases}
$$

Lemma 6 (Handle-slide relation).

$$
X_{m+1} * S_{k}(y)+t^{-4} X_{m} * S_{k}(y)+t^{-2} x^{2} S_{k}(y)=0 .
$$

Proof. The relation is obtained by noting that there is no interaction between the knots $s l_{b}\left(X_{m+1}\right)$, defined in the proof of Lemma 3 and the skein $S_{k}(y)$

Combining the general term in Lemma 5 and the handle slide relation in Lemma 6 , we obtain all the relations reducing $S_{k}(y)$, where $k \geq m+1$, to a skein with lower degree.

Theorem 5. For $k \geq 0$,

$$
\begin{aligned}
& -t^{4 k+4} S_{m+k+2}(y)-t^{4 k+2}\left(t^{-4}-t^{4}+S_{2}(x)\right) S_{m+k+1}(y) \\
& -t^{4 k}\left(t^{-4}-1+\left(t^{-4}+1-t^{4}\right) S_{2}(x)\right) S_{m+k}(y) \\
& -t^{4 k-2}\left(t^{-8}+\left(2 t^{-4}-1-t^{4}\right) S_{2}(x)\right) S_{m+k-1}(y) \\
& +t^{-4 k}\left(t^{8}+\left(2 t^{4}-1-t^{-4}\right) S_{2}(x)\right) S_{m-k}(y) \\
& +t^{-4 k-2}\left(t^{4}-1+\left(t^{4}+1-t^{-4}\right) S_{2}(x)\right) S_{m-k-1}(y) \\
& +t^{-4 k-4}\left(t^{4}-t^{-4}+S_{2}(x)\right) S_{m-k-2}(y)+t^{-4 k-6} S_{m-k-3}(y) \\
& +t^{-2 m-2} x^{2} S_{k+1}(y)+t^{-2 m-2}\left(t^{2}-t^{-2}\right) x^{2} S_{k}(y)+t^{-2 m-2} x^{2} S_{k-1(y)} \\
& +t^{2} \sum_{i=-k+1}^{k-2} \psi_{i-k}(x) t^{4 i} S_{n+i}(y)=0
\end{aligned}
$$

where

$$
\psi_{j}(x)= \begin{cases}t^{4}-t^{-8}+\left(-2 t^{-4}+t+t^{4}\right) S_{2}(x), & \text { if } j \text { is odd } \\ t^{6}-t^{-6}+\left(2 t^{2}-t^{-2}-t^{-6}\right) S_{2}(x), & \text { if } j \text { is even }\end{cases}
$$




\section{Application to the CHARACter VARIETy of The $m$-TWist KnOt}

Recall the following theorem.

Theorem 6 (Bullock [B1], Przytycki-Sikora [PS]). For any compact orientable 3manifold $M$, there exists a surjective homomorphism $\Phi$ as algebra

$$
\Phi: \mathcal{K}_{-1}(M) \rightarrow \chi\left(\pi_{1}(M)\right)
$$

defined by $\Phi(K):=-t_{[K]}, \Phi\left(K_{1} \sqcup \cdots \sqcup K_{i}\right):=\prod_{j=1}^{i} \Phi\left(K_{i}\right)$, where $[K]$ is an element of $\pi_{1}(M)$ represented by the knot $K$ with an unspecified orientation. Moreover the kernel of $\Phi$ is the nilradical $\sqrt{0}$.

According to the above theorem, $R_{m}(-1)$ has information of the defining polynomial of the character variety $X\left(\pi_{1}\left(E_{K_{m}}\right)\right)$, that is.

$$
\chi\left(\pi_{1}\left(E_{K_{m}}\right)\right)=\mathcal{K}_{-1}\left(E_{K_{m}}\right) / \sqrt{0}=\mathbb{C}[x, y] / \sqrt{\left\langle R_{m}(-1)\right\rangle} .
$$

Note that taking the radical of a principal ideal corresponds to getting rid of the multiplicity of each irreducible factor in the irreducible decomposition of the generator. Hence the generator of $\sqrt{\left\langle R_{m}(-1)\right\rangle}$, which ideal is also principal, has the same zeros as $R_{m}(-1)$. In this sense we can consider the polynomial $R_{m}(-1)$ as the defining polynomial of $X\left(E_{K_{m}}\right)$. Using Maple, we can find the following factorizations of $R_{m}(-1)$ over $\mathbb{Q}$ :

$$
\begin{aligned}
& R_{0}(-1)=y+2, \\
& R_{1}(-1)=(y+2)\left(y+x^{2}-1\right), \\
& R_{2}(-1)=(y+2)\left(y^{2}+x^{2} y-y+x^{2}-1\right), \\
& R_{3}(-1)=(y+2)\left(y^{3}+x^{2} y^{2}-y^{2}-2 y+x^{2} y+1\right), \\
& R_{4}(-1)=(y+2)\left(y^{4}+x^{2} y^{3}-y^{3}-3 y^{2}+x^{2} y^{2}-x^{2} y+2 y+1\right), \\
& R_{5}(-1)=(y+2)\left(y^{5}+x^{2} y^{4}-y^{4}-4 y^{3}+x^{2} y^{3}-2 x^{2} y^{2}+3 y^{2}+3 y-x^{2} y+x^{2}-1\right) .
\end{aligned}
$$

A more general fact is true.

Lemma 7. For any non-negative integer $m$, the minimal relation $R_{m}(-1)$ has the following decomposition:

$$
(y+2)\left(S_{m}(y)-S_{m-1}(y)+x^{2} \sum_{i=0}^{m-1} S_{i}(y)\right) .
$$

Moreover, the factor $S_{m}(y)-S_{m-1}(y)+x^{2} \sum_{i=0}^{m-1} S_{i}(y)$ is irreducible over $\mathbb{Q}$.

Proof. The first statement can be shown by using the properties of the Chebyshev polynomial $S_{m}$. The second statement can be proved by a result on the trace field shown by J. Hoste and P. Shanahan [HS]. Let us introduce the notation:

$$
\widetilde{R}_{m}(x, y):=S_{m}(y)-S_{m-1}(y)+x^{2} \sum_{i=0}^{m-1} S_{i}(y) .
$$

In the case of $m=0,1$, (that is the case of the unknot and the right-handed trefoil which are non-hyperbolic knots), it was observed above. Next, consider the case 
where $m \geq 2$. Then the twist knot $K_{m}$ is hyperbolic. Hence there exists the discrete faithful representation

$$
\rho_{0}: \pi_{1}\left(E_{K_{m}}\right) \rightarrow S L(2, \mathbb{C})
$$

of $\pi_{1}\left(E_{K_{m}}\right)$. By Theorem 6 , we can regard the skeins $x$ and $y$ as the functions $-t_{x}$ and $-t_{y}$, respectively. Here $x$ is a meridional skein so we can assume that

$$
x\left(\rho_{0}\right)=-t_{x}\left(\rho_{0}\right)= \pm 2, y\left(\rho_{0}\right)=-t_{y}\left(\rho_{0}\right)=-2 \text { or } \alpha,
$$

where $\alpha$ is a solution of $\widetilde{R}_{m}( \pm 2, y)=0$ over $\mathbb{C}$.

Remark 1. The diagram in Figure 1 is alternating and irreducible, so the minimal crossing number of $K_{m}$ is exactly $m+2$ (see [K, M, T] for details).

Now, by Corollary 1 in $[\mathrm{HS}]$ and Remark 1 , the extension field $\mathbb{Q}\left(t_{\gamma}\left(\rho_{0}\right): \gamma \in\right.$ $\pi_{1}\left(E_{K_{m}}\right)$ ) over $\mathbb{Q}$, called the trace field of $K_{m}$, has degree $m$. Namely,

$$
\left[\mathbb{Q}\left(t_{\gamma}\left(\rho_{0}\right): \gamma \in \pi_{1}\left(E_{K_{m}}\right)\right): \mathbb{Q}\right]=m .
$$

Here it can be shown that $\mathbb{Q}\left(t_{\gamma}\left(\rho_{0}\right): \gamma \in \pi_{1}\left(E_{K_{m}}\right)\right)$ is simple extension, that is,

$$
\mathbb{Q}\left(t_{\gamma}\left(\rho_{0}\right): \gamma \in \pi_{1}\left(E_{K_{m}}\right)\right)=\mathbb{Q}(\alpha) .
$$

(Refer to $[\mathrm{CR}, \mathrm{NR}]$ for details). If $y=-2$, then $\mathbb{Q}(-2)=\mathbb{Q}$, a contradiction. Therefore $y$ should be equal to $\alpha$, which is a solution of $\widetilde{R}_{m}( \pm 2, y)$ with $\operatorname{deg}_{y}=m$. Hence $\widetilde{R}_{m}( \pm 2, y)$ must be irreducible over $\mathbb{Q}$. It is not so hard to see that if $\widetilde{R}_{m}(x, y)$ is reducible, then so is $\widetilde{R}_{m}( \pm 2, y)$. These two facts complete the proof.

It follows from the definition of the character variety that $X(M)$ for any compact orientable 3-manifold $M$ is defined over $\mathbb{Q}$ (in fact over $\mathbb{Z}$ ). Hence by Lemma 7, we obtain:

Theorem 7. Consider the character ring $\chi^{\mathbb{Q}}\left(\pi_{1}\left(E_{K_{m}}\right)\right)$ and the $K B S A \mathcal{K}_{-1}^{\mathbb{Q}}\left(E_{K_{m}}\right)$ whose coefficient fields are $\mathbb{Q}$. Then $\mathcal{K}_{-1}^{\mathbb{Q}}\left(E_{K_{m}}\right)$ has trivial nilradical. Therefore the following holds:

$$
\chi^{\mathbb{Q}}\left(\pi_{1}\left(E_{K_{m}}\right)\right)=\mathbb{Q}[x, y] /\left\langle R_{m}(-1)\right\rangle .
$$

\section{ACKNOWLEDGEMENTS}

The second author would like to thank Professor Makoto Sakuma for the helpful comments and Professor Mitsuyoshi Kato for his encouragement.

\section{REFERENCES}

[B1] D. Bullock: Rings of $S L_{2}(\mathbb{C})$-characters and the Kauffman bracket skein module, Comment. Math. Helv. 72 (1997), 521-542.

[B2] D. Bullock: The $(2, \infty)$-skein module of the complement of a $(2,2 p+1)$-torus knot, J. Knot Theory Ramifications 4 (1995), 619-632.

[BL] D. Bullock and W. LoFaro: The Kauffman bracket skein module of a twist knot exterior, preprint.

[CR] P. J. Callahan and A. W. Reid: Hyperbolic structure on knot complements, Chaos, Solitons and Fractals 9 (1998), 705-738.

[CCGLS] D. Cooper, M. Culler, H. Gillet, D. Long and P. Shalen: Plane curves associated to character varieties of 3-manifolds, Invent. Math. 118 (1994), 47-84.

[CL1] D. Cooper and D. Long: Remarks on the A-polynomial of a knot, J. Knot Theory Ramifications 5 (1996), 609-628. 
[CL2] D. Cooper and D. Long: Representation theory and the A-polynomial of a knot, Chaos Solitons Fractals 9 (1998), 749-763.

[CS] M. Culler and P. Shalen: Varieties of group presentations and splittings of 3-manifolds, Ann. of Math. (2) 117 (1983), 109-146.

[FG] C. Frohman and R. Gelca: Skein modules and the noncommutative torus, Trans. Amer. Math. Soc. 352 (2000), 4877-4888.

[FGL] C. Frohman, R. Gelca and W. LoFaro: The A-polynomial from the noncommutative viewpoint, Trans. Amer. Math. Soc. 354 (2001), 735-747.

[GL] S. Garoufalidis and T. T. Q. Le: The colored Jones function is q-holonomic, preprint.

[G1] R. Gelca: Noncommutative trigonometry and the A-polynomial of the trefoil knot, Math. Proc. Cambridge Philos. Soc. 133 (2002), 311-323.

[G2] R. Gelca: On the relation between the A-polynomial and the Jones polynomial, Proc. Amer. Math. Soc. 130 (2001), 1235-1241.

[GS1] R. Gelca and J. Sain: The noncommutative A-ideal of a $(2,2 p+1)$-torus knot determines its Jones polynomial, J. Knot Theory Ramifications, 2(2003), 187-201.

[GS2] R. Gelca and J. Sain: The computation of the noncommutative generalization of the Apolynomial of the figure-eight knot, J. Knot Theory Ramifications, 6(2004) 785-808.

[HP] J. Hoste and J. Przytycki: The $(2, \infty)$-skein module of lens spaces; a generalization of the Jones polynomial, J. Knot Theory Ramifications 2 (1993), 321-333.

[HS] J. Hoste and P. Shanahan: Trace fields of twist knots, J. Knot Theory Ramifications 10 (2001), 625-639.

[K] H. Kauffman: State models and the Jones polynomial, Topology 26 (1987), 395-407.

[L] T.T.Q. Le: The colored Jones polynomial and the A-polynomial of two-bridge knots, math.GT/040752.

[M] K. Murasugi: Jones polynomials and classical conjectures in knot theory, Topology 26 (1987), 187-194.

[N] F. Nagasato: An approach to the A-polynomial of $(2,2 p+1)$ torus knots from the FrohmanGelca-Lofaro theory, J. Knot Theory Ramif., to appear.

[NR] W. Neumann and A. Reid: Arithmetic of hyperbolic manifolds, Topology2̆01890, de Gruyter (1992), 17-26.

[P1] J. Przytycki: Fundamentals of Kauffman bracket skein module, Kobe J. Math. 16 (1999), no. 1, 45-66.

[P2] J. Przytycki: Skein modules of 3-manifolds, Bull. Pol. Acad. Sci. 39 (1991), 91-100.

[PS] J. H. Przytycki and A. Sikora, Skein algebra of a group, Banach Center Publ. 42.

[T] M. Thistlethwaite: A spanning tree expansion of the Jones polynomial, Topology 26 (1987), 297-309.

Department of Mathematics and Statistics, Texas Tech University, Lubbock, Texas 79409, USA and Institute of Mathematics of the Romanian Academy, Bucharest, ROMANIA

E-mail address: rgelca@math.ttu.edu

Faculty of Mathematics, Kyushu University, Hakozaki 6-10-1, Higashi-Ku, Fukuoka, 812-8581, JAPAN

E-mail address: fukky@math.kyushu-u.ac.jp 